\title{
Molecular Magnetic Quantum Dots in Multivalent Metal Cluster Compounds
}

\author{
J. Sinzig and L. J. de Jongh \\ Kamerlingh Onnes Laboratory, Leiden University, P.O. Box 9506, NL 2300 RA Leiden, The Netherlands \\ A. Ceriotti and R. della Pergola \\ Dipartimento di Chimica Inorganica e Metallorganica, Via G. Venezian 21, 20133 Milano, Italy \\ G. Longoni \\ Dipartimento di Chimica Fisica ed Inorganica, Universitá di Bologna, Viale del Risorgimento 4, 40136 Bologna, Italy \\ M. Stener, K. Albert, and N. Rösch \\ Lehrstuhl für Theoretische Chemie, Technische Universität München, Lichtenbergstraße 4, 85747 Garching, Germany
}

(Received 24 February 1998)

\begin{abstract}
In most magnetic molecular clusters studied so far, localized moments of metal ions are coupled by superexchange interactions via ligand atoms to yield the total moment of the cluster, which is basically a piece of a magnetic insulator. Here we present an experimental and theoretical study of another form of molecular magnetism, arising from unfilled molecular orbitals delocalized over the entire metal cores of molecular metal clusters. These cores thus act as nanosize quantum dots, in which the metal valence electrons are confined. [S0031-9007(98)07157-9]
\end{abstract}

PACS numbers: 73.20.Dx, 75.50.Tt

Magnetic clusters are drawing increasing attention as the ultimate lower size limit for magnetic nanoparticles [1]. Fundamental scientific questions at stake are the quantum-size effects on the thermodynamic properties [2], and the phenomenon of macroscopic magnetic quantum tunneling [3]. Molecular cluster compounds play a crucial role in these studies, since they offer the unique possibility to investigate macroscopically large assemblies of identical (monodisperse) clusters, using well-known techniques of solid state physics and chemistry [1,4,5]. For these well-defined, stoichiometric chemical compounds, the clusters are cores of macromolecules, separated from one another by shells of ligand molecules coordinated to the cluster surface. In metal cluster compounds [4,5], the cluster core is composed of metal atoms only, bound to one another by direct metal-metal bonds. In ionic metal clusters [1], on the other hand, the cluster framework consists of metal ions linked by intervening nonmetal ions. Thus, the clusters in these two classes of materials can be seen as small pieces of bulk metal and of bulk insulator, respectively.

Most of the molecular magnetic clusters studied, for example, the compound $\mathrm{Mn}_{12} \mathrm{Ac}$, belong to the second class $[1,3]$. The magnetism of the cluster then basically arises from unfilled atomic shells, and the moments of the unpaired electrons are localized on metal atoms and are coupled through superexchange via intervening nonmetal ions. The net magnetic moment of the cluster arises from interacting atomic moments, where the interactions can be ferro- or antiferromagnetic.

In this Letter we address a form of molecular magnetism in which the magnetic behavior cannot be rationalized in terms of an atomic property. As we shall show, there exist several molecular metal cluster compounds in which unpaired electrons occupy molecular orbitals delocalized over the whole metal framework formed by direct metal-metal bonds. The magnetism is due to unfilled, delocalized cluster orbitals, and is a molecular analog of the magnetism of unfilled atomic orbitals, the unpaired electrons being extended over the much larger volume of the (macro)molecule instead of that of the atom.

We focus our attention on the multivalent metal carbonyl cluster molecules $\left[\mathrm{Pt}_{3} \mathrm{Fe}_{3}(\mathrm{CO})_{15}\right]^{n-}\left(\mathbf{1}^{n-}\right.$ for short $)$ and $\left[\mathrm{Ag}_{13}\left\{\mathrm{Fe}(\mathrm{CO})_{4}\right\}_{8}\right]^{n-}\left(\mathbf{2}^{n-}\right)$ occurring in $\mathbf{1}^{n-}\left[\mathrm{PPh}_{4}^{+}\right]_{n}(n=$ $1,2)$ and $2^{n-}\left[\mathrm{N}\left(\mathrm{PPh}_{3}\right)_{2}{ }^{+}\right]_{n}(n=3,4)$, the structures of which are shown in Fig. 1 [6-9]. Depending on the valency, these materials are either nonmagnetic or carry an unpaired spin $\frac{1}{2}$ on each cluster molecule. As we shall show both experimentally and theoretically, the origin of this spin $\frac{1}{2}$ is a singly occupied highest molecular orbital (HOMO) that is delocalized over the entire cluster core.

Magnetic measurements in the temperature range $0.1-$ $300 \mathrm{~K}$ and in magnetic fields up to $5 \mathrm{~T}$ were made using SQUID magnetometry. In Fig. 2 we show susceptibility data on powder samples plotted as $\chi$ versus $T$ for $\mathbf{1}^{-}\left[\mathrm{PPh}_{4}\right]$ and $2^{4-}\left[\mathrm{N}\left(\mathrm{PPh}_{3}\right)_{2}\right]_{4}$, which both contain one unpaired electron. Perfect Curie behavior (solid curves through the data), corresponding to $1 \mathrm{spin} /$ cluster, is found down to $20 \mathrm{~K}$ for the $\mathrm{Pt}_{3} \mathrm{Fe}_{3}$ system and down to $0.1 \mathrm{~K}$ for the $\mathrm{Ag}_{13} \mathrm{Fe}_{8}$ compound. For $\mathrm{Pt}_{3} \mathrm{Fe}_{3}$ the susceptibility shows a maximum below $1 \mathrm{~K}$, which we attribute to antiferromagnetic interactions between spins on neighboring clusters. The solid curve through these data is a fit to the antiferromagnetic, $S=\frac{1}{2}$, Heisenberg chain model [10], yielding an exchange constant $J / k_{B}=$ $-0.46 \mathrm{~K}\left(\mathcal{H}=-2 J \sum_{i, j} \overrightarrow{\mathrm{S}}_{i} \cdot \overrightarrow{\mathrm{S}}_{j}\right)$. The packing of the 
a)

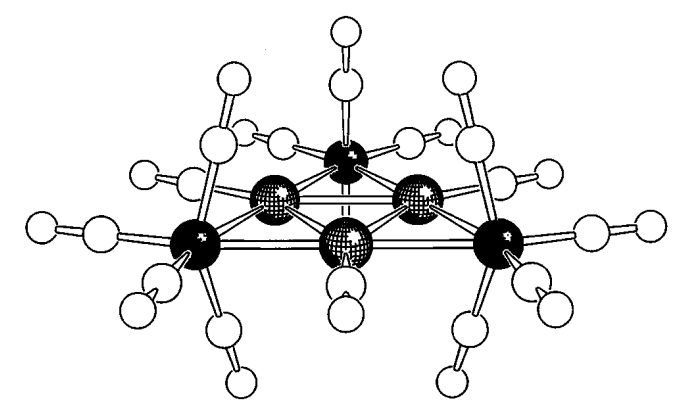

b)

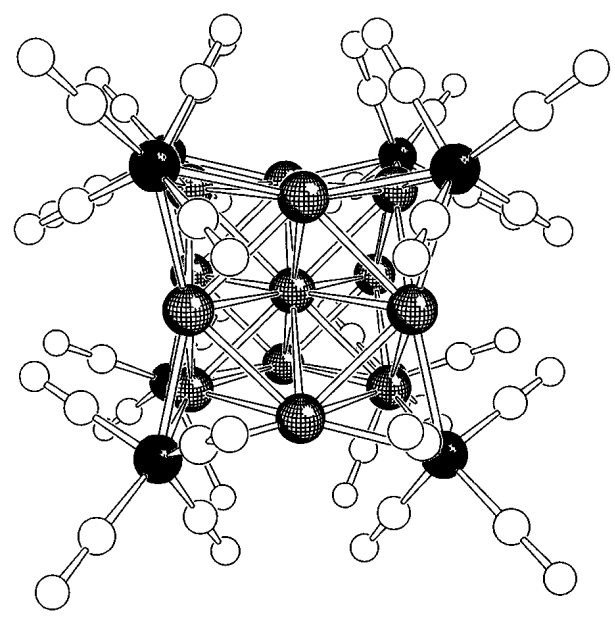

FIG. 1. (a) Structure of the cluster $\mathbf{1}^{-},\left[\mathrm{Pt}_{3} \mathrm{Fe}_{3}(\mathrm{CO})_{15}\right]^{-}$. Pt shaded; Fe black. (b) Averaged experimental geometry of the cluster anion $2^{4-},\left[\mathrm{Ag}_{13}\left\{\mathrm{Fe}(\mathrm{CO})_{4}\right\}_{8}\right]^{4-}$. Ag shaded; Fe black.

cluster molecules in the crystal is indeed found to be predominantly in chains, supporting the interpretation in terms of quasi-one-dimensional magnetic behavior. The specific heat of this compound (not shown) likewise confirms the magnetic chain behavior; it has a magnetic contribution with a broad maximum at about $0.7 \mathrm{~K}$ that can also be described by this theoretical model. The absence of appreciable magnetic interactions between the $\mathrm{Ag}_{13} \mathrm{Fe}_{8}$ clusters may be similarly understood by the packing of the clusters in the crystal; in this compound they are widely separated by ligands and cations (closest distances of about $6.7 \AA$ ).

Also the magnetization data in the range $3-100 \mathrm{~K}$ (cf. Fig. 3) for both compounds show the $S=\frac{1}{2}$ paramagnetic behavior. They can be well fitted to the Brillouin curve, with $S=\frac{1}{2}$ and average $g$ values $g=\left(\frac{1}{3} g_{\|}^{2}+\frac{2}{3} g_{\perp}^{2}\right)^{1 / 2}$, where the values for $g_{\|}$and $g_{\perp}$ were determined independently by ESR as $g_{\|}=2.24, g_{\perp}=2.06$ and $g_{\|}=2.0465$, $g_{\perp}=1.937$, for the $\mathrm{Pt}_{3} \mathrm{Fe}_{3}$ and the $\mathrm{Ag}_{13} \mathrm{Fe}_{8}$ compound, respectively (the same average $g$ values were used in the susceptibility fits). In both cases the hyperfine splitting of the ESR line is due to the interaction of the unpaired elec-
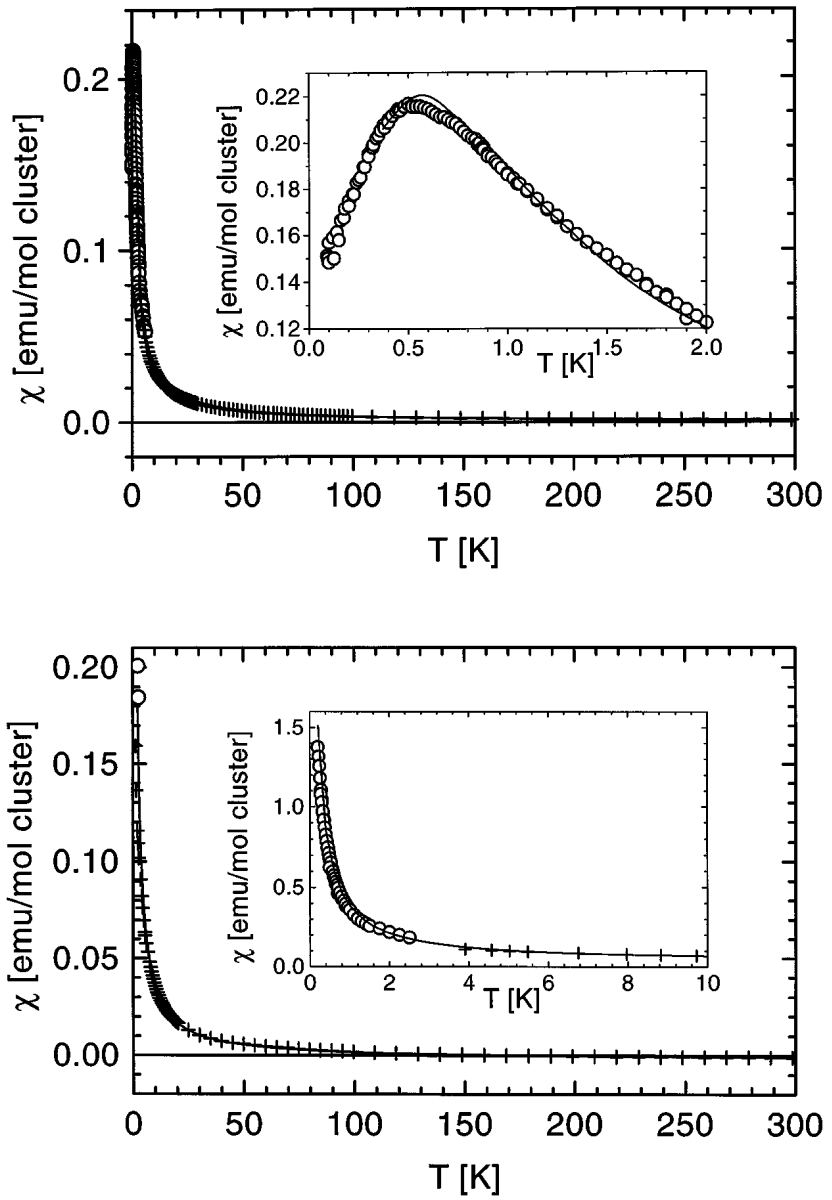

FIG. 2. Top: Magnetic susceptibility of $\mathbf{1}^{-}\left[\mathrm{PPh}_{4}\right]$ between 0.1 and $300 \mathrm{~K}$. Solid line through data in inset is the fit to the antiferromagnetic chain model. Bottom: susceptibility of $2^{4-}\left[\mathrm{N}\left(\mathrm{PPh}_{3}\right)_{2}\right]_{4}$. Inset shows data below $2.5 \mathrm{~K}$.

tron spin with the nuclear spins of Pt and Ag, respectively $[6,8]$ (isotope ${ }^{57} \mathrm{Fe}$ features a nuclear spin, but with only $2 \%$ abundance, it cannot be detected). The ESR spectra thus point to a substantial amount of spin density at the nonmagnetic metal sites, in good agreement with the calculated spin density maps presented below. This illustrates, in fact, that the extent of delocalization can be inferred quite well from measurements of hyperfine couplings.

Magnetic measurements (not shown) on the nonmagnetic versions, $\mathbf{1}^{2-}\left[\mathrm{PPh}_{4}\right]_{2}$ and $\mathbf{2}^{3-}\left[\mathrm{N}\left(\mathrm{PPh}_{3}\right)_{2}\right]_{3}$, confirm that these valencies correspond to filled molecular orbitals. The compounds are intrinsically nonmagnetic, except for traces of the magnetic versions, which appear to be present in varying small amounts, depending on the method of preparation, aging of the sample, etc. The identification of the weak magnetic signals as originating from the magnetic counterparts could be done unambiguously from the ESR spectra, which could always be traced to the magnetic versions (absolute calibration of the ESR signal was performed and proved to agree with the amount of impurity spins found from the magnetization). 

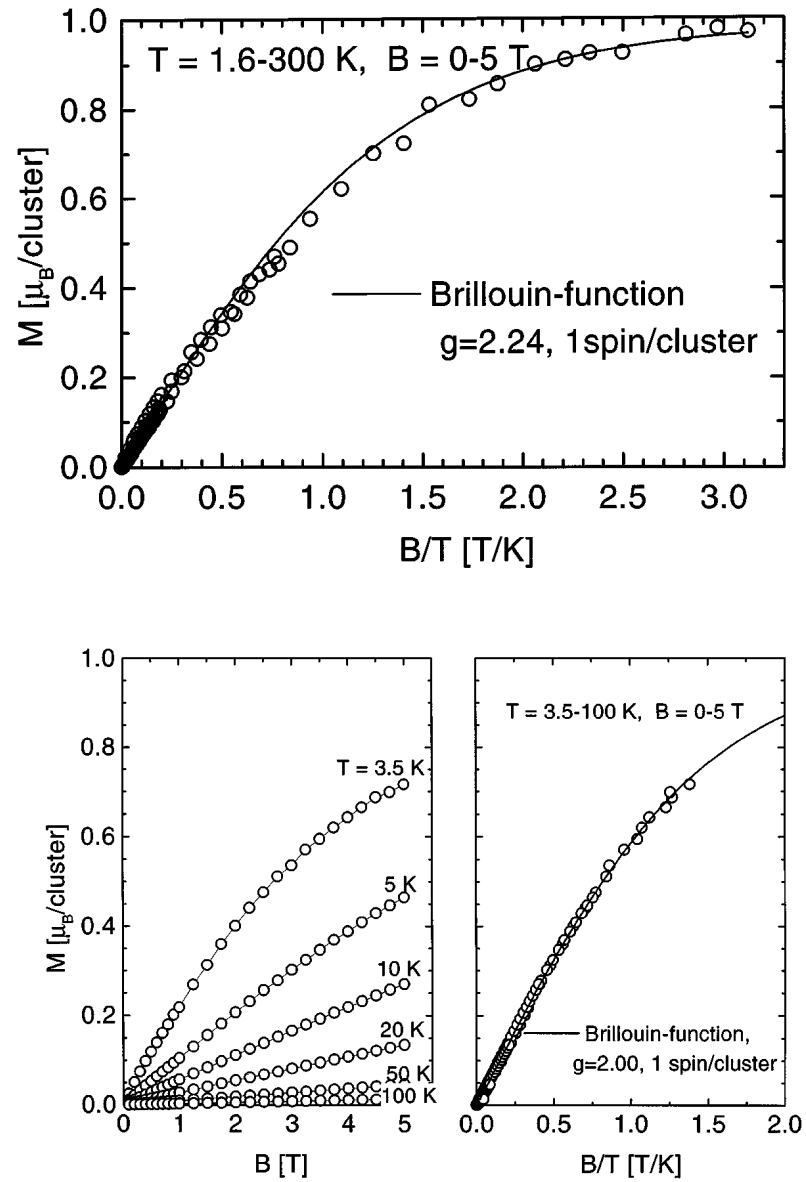

FIG. 3. Magnetization curves of $\mathbf{1}^{-}\left[\mathrm{PPh}_{4}\right]$ (top panel) and $\mathbf{2}^{4-}\left[\mathrm{N}\left(\mathrm{PPh}_{3}\right)_{2}\right]_{4}$ (lower panel). Data are plotted versus the magnetic field $B$ or $B / T$ and fit the Brillouin function with one spin per cluster.

In Fig. 4 we show spin density maps for the magnetic versions of these materials as predicted by density functional calculations, using a self-consistent, scalarrelativistic linear combination of Gaussian-type orbitals method [11] with a gradient corrected exchangecorrelation functional [12]. Spin-unrestricted calculations were performed to describe open-shell configurations. Atomic charges and orbital localizations were computed with a Mulliken analysis. The optimized geometries of the clusters $\mathbf{1}^{n-}(n=0,1,2)$ agree quite well with the experimentally determined structures, especially with regards to the metal-metal distances [13]. For the clusters $\mathbf{2}^{n-}$ an averaged experimental geometry was used, idealized to $\mathrm{O}_{h}$ symmetry [14].

The spin density maps in Fig. 4 refer to the cluster anions $\mathbf{1}^{-}$and $\mathbf{2}^{4-}$. In each case, the HOMO is nondegenerate, singly occupied, and well-separated by a HOMO-LUMO gap of the order of $1 \mathrm{eV}$ from the lowest unoccupied orbital (LUMO), but also by about the same energy from the next lower, completely filled molecular orbital. In the nonmagnetic anion $\mathbf{1}^{2-}$, the additional electron fills the HOMO level completely, as expected. a)

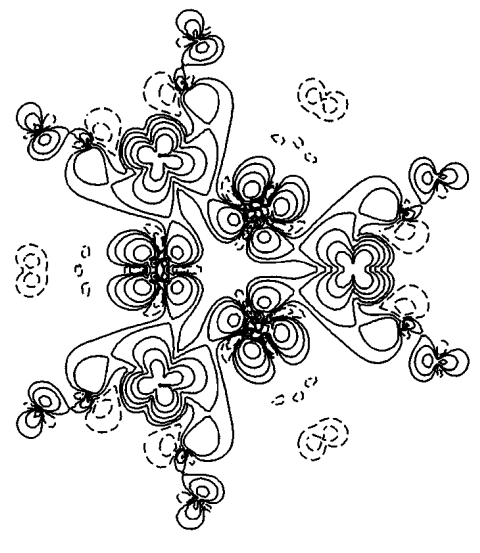

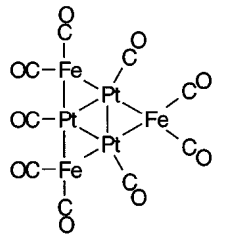

b)

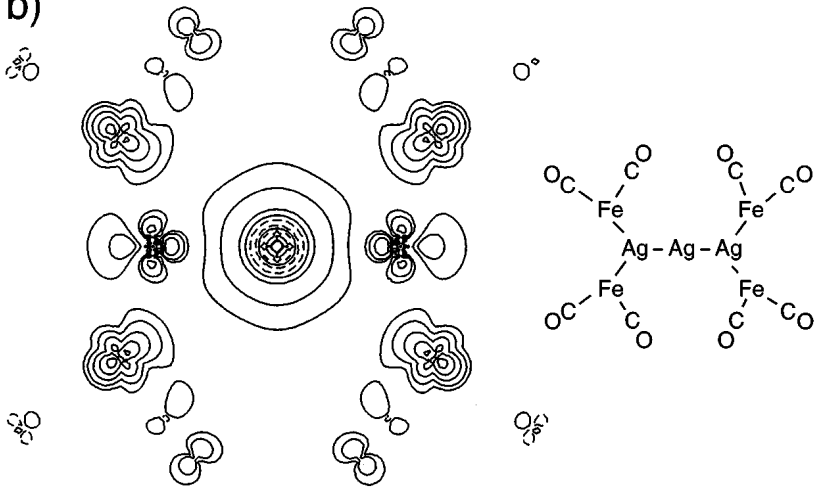

FIG. 4. Spin density contour plots of (a) $\mathbf{1}^{-}$(in the plane which contains the $\mathrm{Fe}$ and $\mathrm{Pt}$ atoms and nine equatorial $\mathrm{CO}$ ligands) and (b) $\mathbf{2}^{4-}$ (in a plane which contains the central and two peripheral $\mathrm{Ag}$ atoms, four $\mathrm{Fe}$ atoms, and eight $\mathrm{CO}$ groups). Solid and dashed contour lines indicate positive and negative values, respectively, at values $10^{-n / 2}$ a.u. $(n=3-7)$.

On the other hand, in the nonmagnetic anion $2^{3-}$ the HOMO of the magnetic cluster is unoccupied. In both magnetic clusters, the spin density is largely determined by the HOMO; however, noticeable deviations are found [13]. As seen from Fig. 4, the spin density is delocalized not only over the $\mathrm{Fe}$ atoms, but also over the $\mathrm{Ag}$ atoms. In the $\mathrm{Pt}_{3} \mathrm{Fe}_{3}$ cluster, about $18 \%$ population of the spin density is localized on the $3 \mathrm{Pt}$ atoms and about $70 \%$ on the $3 \mathrm{Fe}$ atoms, confirming that the magnetism is not carried by electrons in localized $(\mathrm{Fe})$ atomic orbitals, but in delocalized cluster molecular orbitals. For the $\mathrm{Ag}_{13} \mathrm{Fe}_{8}$ cluster, the ESR data [8] showed that $25 \%$ of the spin density is located on the central $\mathrm{Ag}$ atom, whereas the 12 peripheral $\mathrm{Ag}$ atoms carry negligible spin $(\simeq 1 \%)$. This would imply an even larger spin density on the central $\mathrm{Ag}$ atom than on each of the $\mathrm{Fe}$ atoms $(\simeq 10 \%)$. The calculated spin density plot shows indeed considerable delocalization of the spin density over the Fe atoms $(\simeq 50 \%$ population on $6 \mathrm{Fe}$ centers) and $\mathrm{Ag}$ atoms $(\simeq 8 \%$ on the central $\mathrm{Ag}$ atom, $\simeq 32 \%$ on the 12 peripheral $\mathrm{Ag}$ centers), in broad agreement with the ESR spectra [15]. 
In the older literature on small organometallic clusters, several reports may already be found on small magnetic metal clusters for which the magnetism was attributed to unpaired electrons delocalized over the metal framework. We mention $\left[\mathrm{Ni}\left(\eta^{5}-\mathrm{C}_{5} \mathrm{H}_{5}\right)\right]_{3}\left(\right.$ tert. $\left.-\mathrm{C}_{5} \mathrm{H}_{9} \mathrm{~N}\right)$ and $\left[\mathrm{Co}\left(\eta^{5}-\mathrm{C}_{5} \mathrm{H}_{5}\right)\right]_{3} \mathrm{~S}_{2}$, with one and two unpaired electrons per cluster, respectively [16,17]. The monocation related to this $\mathrm{Co}_{3} \mathrm{~S}_{2}$ cluster, as occurring in $\left[\mathrm{Co}_{3}\left(\eta^{5}-\mathrm{C}_{5} \mathrm{H}_{5}\right)_{3} \mathrm{~S}_{2}\right]^{+} \mathrm{I}^{-}$, was found to have a single unpaired electron [17]. The delocalized nature is indicated by ESR data on these materials. Another example of a multivalent metal cluster is $\left[\mathrm{Ni}_{6}\left(\eta^{5}-\mathrm{C}_{5} \mathrm{H}_{5}\right)_{6}\right]^{n}$ $(n=0,+1)$, which is reported to be nonmagnetic when neutral, but to carry three unpaired electrons in cationic form [18]. Three unpaired electrons were also found for $\left[\mathrm{Ni}\left(\mathrm{C}_{5} \mathrm{H}_{5}\right)\right]_{4} \mathrm{H}_{3}$ [19]; they have been shown to occupy a spatially almost degenerate set of three cluster orbitals [20]. Although these older results should be further investigated both experimentally and theoretically, they appear to indicate that even a high-spin configuration within the highest lying molecular orbital manifold may result from the concerted action of direct metal-metal exchange, ligand-field interactions from the surrounding ligand shell, and spin-orbit coupling.

In conclusion, we have presented conclusive evidence for a new form of molecular magnetism in which molecular metal clusters act as a potential well in which unpaired delocalized cluster valence electrons are confined. This leads to a magnetism of unfilled molecular electron shells, quite analogously to the traditional magnetism of unfilled atomic shells. We have shown that, for suitable packing, exchange interactions between these magnetic molecular clusters may lead to magnetic ordering phenomena at low temperatures. The major difference with atoms lies, of course, in the sizes of the electron orbitals, which are much larger in the clusters. This may lead to special features that deserve further investigation. In particular, the magnetic properties of such clusters should be susceptible to tuning by molecular design, e.g., by variations of ligands and counterions.

We thank Y. Volokitin for help in the measurements below $1 \mathrm{~K}$, and for stimulating discussions. This work was part of the research program of the Stichting voor Fundamenteel Onderzoek der Materie (FOM), which is supported by the Nederlandse Organisatie voor Wetenschappelijk Onderzoek (NWO). Support of the Fonds der Chemische Industrie and of the European Community under the HCM program is gratefully acknowledged.
[1] See, e.g., D. Gatteschi, A. Caneschi, L. Pardi, and R. Sessoli, Science 265, 1054-1056 (1994).

[2] Y. Volokitin, J. Sinzig, L. J. de Jongh, G. Schmid, M. N. Vargaftik, and I. I. Moiseev, Nature (London) 384, 621623 (1996); S. G. Louie, ibid. 384, 612 (1996).

[3] L. Thomas, F. Lionti, R. Ballou, D. Gatteschi, R. Sessoli, and R. Barbara, Nature (London) 383, 145-147 (1996); J. M. Hernandez, Y.X. Zhang, F. Luis, J. Bartolomé, J. Tejada, and R. Ziolo, Europhys. Lett. 35, 301-306 (1996).

[4] Clusters and Colloids. From Theory to Application, edited by G. Schmid (VCH, Weinheim, Germany, 1994).

[5] Physics and Chemistry of Metal Cluster Compounds, edited by L. J. de Jongh (Kluwer, Dordrecht, 1994) (ISBN 0-7923-2715-2).

[6] G. Longoni, M. Manassero, and M. Sansoni, J. Am. Chem. Soc. 102, 7973-7974 (1980).

[7] S. Gorter (private communication); (to be published).

[8] V.G. Albano, L. Grossi, G. Longoni, M. Monari, S. Mulley, and A. Sironi, J. Am. Chem. Soc. 114, 5708-5713 (1992).

[9] V. G. Albano, F. Calderoni, M. C. Iapalucci, G. Longoni, M. Monari, and P. Zanello, J. Cluster Sci. 6, 107-123 (1995).

[10] J.C. Bonner and M.E. Fisher, Phys. Rev. 135, A640A658 (1964).

[11] N. Rösch, S. Krüger, M. Mayer, and V. A. Nasluzov, in Recent Developments and Applications of Modern Density Functional Theory, edited by J.M. Seminario (Elsevier, Amsterdam, 1996), pp. 497-566.

[12] A. Becke, Phys. Rev. A 38, 3098-3100 (1988); J.P. Perdew, Phys. Rev. B 33, 8822-8824 (1986).

[13] M. Stener, K. Albert, and N. Rösch (to be published).

[14] K. Albert, K. M. Neyman, G. Pacchioni, and N. Rösch, Inorg. Chem. 35, 7370-7376 (1996).

[15] The combined Ag spin density population on the central and the 12 peripheral centers is calculated to $40 \%$ as compared to $\simeq 37$ in experiment. Inspection of the HOMO contour plot suggests that this large difference is likely due to well-known limitations of the Mulliken procedure.

[16] S. Otsuka, A. Nakamura, and T. Yoshida, Liebigs Ann. Chem. 719, 54-60 (1968).

[17] P.D. Frisch and L. F. Dahl, J. Am. Chem. Soc. 94, 50825084 (1972).

[18] M. S. Paquette and L.F. Dahl, J. Am. Chem. Soc. 102, $6621-6623$ (1980).

[19] J. Müller, H. Dorner, G. Huttner, and H. Lorenz, Angew. Chem. Int. Ed. Engl. 12, 1005 -1006 (1973).

[20] R. Hoffmann, B. E. R. Schilling, R. Bau, H. D. Kaesz, and D. M. P. Mingos, J. Am. Chem. Soc. 100, 6088-6093 (1978). 